\title{
Dietary Consumption of Virgin Coconut Oil Ameliorates Lipid Profiles in Diabetic Rats
}

\author{
A. M. Akinnuga, ${ }^{1}$ S. O. Jeje, ${ }^{1}$ O. Bamidele, ${ }^{2}$ and V. E. Sunday ${ }^{1}$ \\ ${ }^{1}$ Department of Human Physiology, Cross River University of Technology, Okuku Campus, Yala, Cross River State, Nigeria \\ ${ }^{2}$ Department of Physiology, Bowen University, Iwo, Osun State, Nigeria \\ Correspondence should be addressed to A. M. Akinnuga; akinnugaakinjide@yahoo.com
}

Received 24 August 2014; Revised 12 September 2014; Accepted 14 September 2014; Published 29 September 2014

Academic Editor: Gary Lopaschuk

Copyright (c) 2014 A. M. Akinnuga et al. This is an open access article distributed under the Creative Commons Attribution License, which permits unrestricted use, distribution, and reproduction in any medium, provided the original work is properly cited.

\begin{abstract}
Virgin coconut oil (VCO) is a saturated fat with promising antidiabetic properties but its ameliorative effect on lipid profiles in diabetics is rarely reported. Therefore, in this study, a total of fifteen (15) male rats weighing 200-250 g were divided into 3 experimental groups $(n=5)$. Group I (control) and Group II (diabetic control group) were fed a normal rat chow while Group III (diabetic test group) was fed a 10\% VCO diet for 3 weeks. Group II and Group III were made diabetic by intraperitoneal injection of $150 \mathrm{mg} / \mathrm{kg}$ of alloxan. After 72 hours of injection, blood glucose was tested to confirm diabetes mellitus. After 3 weeks, the animals were sacrificed to collect blood samples for lipid profile analysis. The results showed a significant increase in concentrations of triglyceride, total cholesterol, low density lipoprotein, and very low density lipoprotein and decrease in concentration of high density lipoprotein in Group II when compared to Group I. Also, the concentrations of triglyceride, total cholesterol, low density lipoprotein, and very low density lipoprotein except high density lipoprotein significantly reduced in Group III when compared to Group II $(P<0.01,0.001)$. VCO consumption can be claimed to ameliorate lipid levels in diabetes mellitus.
\end{abstract}

\section{Introduction}

Diabetes mellitus is the most common serious metabolic disease in human with a hall mark of an elevated blood glucose concentration caused by a number of biochemical and physiological alterations [1]. It is a chronic menace to the entire human race without any cogent cure except management over some decades now.

It is estimated that about 3.2 million diabetes related deaths are reported annually and this condition is worst in developing countries where the number of people afflicted is expected to increase by $150 \%$ by the year 2030 [2]. However, it is a metabolic disorder in which the level of blood glucose is persistently raised above normal range $(80-100 \mathrm{mg} / \mathrm{dL}$ ) due to oxidative stress which induces insulin resistance in the peripheral tissues (type 2 diabetes mellitus) and impairs insulin secretion from pancreatic $\beta$-cells (type 1 diabetes mellitus).

Furthermore, diabetes mellitus is characterized by excessive disturbance of carbohydrates, proteins and lipid metabolism, and thickening of capillary basement membrane throughout the body leading to long term complications such as renal dysfunction, neuropathy, retinopathy, and cardiovascular problems. Interestingly, among these complications, cardiovascular problems (especially atherosclerosis and coronary artery disease) are due to altered plasma lipid and lipoprotein levels which result from an altered intermediary metabolism of serum lipids and lipoproteins [3].

In addition, the consumption of saturated fatty acid (SFA) including rich dietary vegetable oils such as coconut oil and palm oil has before now been discouraged and side effects of virgin coconut oil consumption have been reported. This is due to the belief that excess consumption of saturated fats under diabetes mellitus condition can accelerate the atherosclerotic process but most of the recent investigations conducted in animals as well as human beings contradict claims that coconut oil increases the risk of atherosclerosis and heart disease $[4,5]$. 
Also, several studies looking at populations where virgin coconut oil is consumed in larger quantities have not found higher rates of coronary artery disease or elevated cholesterol level that have alliance with diabetes and other ailments [6].

Coconut oil is a source of tocotrienols, capric acid, caproic acid, and lauric acid which are natural antioxidants. These substances act as scavengers of damaging oxygen free radicals that have been suggested to play an important role in aging, atherosclerosis, cancer, and diabetes mellitus $[7,8]$.

Therefore, due to the above background, virgin coconut oil (VCO) is promoted as a dietary supplement for people with various ailments such as diabetes mellitus. However, there have been few formal scientific studies to validate its health benefits; thus the need to investigate the ameliorative effect of dietary consumption of coconut oil in diabetics where lipid profile is abnormal using animal model of type 1 diabetes mellitus as case study is inevitable.

\section{Materials and Methods}

\subsection{Plant}

2.1.1. Preparation of Virgin Coconut Oil (VCO). Dry coconuts were purchased from Gakem, Okuku, and Bekwarra markets in the northern part of Cross River State, Nigeria. The nuts were broken manually; its meat scrapped from the shell and cut into a small piece using a sharp paring knife. The cut pieces were grinded in a grinding machine into viscous slurry and, thereafter, squeezed through cheese cloth to obtain coconut milk which was put into glass jars. The glass jars containing the squeezed coconut milk were left for at least 24 hours to allow the coconut milk and oil to separate into a layer of curd which appears at the top of the jars. The jars were refrigerated for 48 hours so that the curd could harden. Thereafter, the curd was scooped out and discarded leaving the pure virgin oil in the jars. The obtained VCO was decanted into a bottle with a plastic screw cap and stored at room temperature for use in the present study.

However, the preparation of the $10 \%$ virgin coconut oil diet was done by mixing and mashing $100 \mathrm{~g}$ of virgin coconut oil with $900 \mathrm{~g}$ of normal rat chow until there was homogeneity.

The preparation of the $10 \%$ virgin coconut oil meal was done on regular demand [9].

2.2. Experimental Animals. Fifteen (15) male Wistar rats weighing 200-250 g were used in this study. The rats were purchased at the animal house of the Department of Human Physiology, Faculty of Basic Medical Sciences, Cross River University of Technology (CRUTECH), Okuku Campus, Nigeria. The animals were kept in cages with suitable temperature, humidity, water, and normal rat chow for 2 weeks to acclimatize.

2.3. Experimental Procedure. This work was carried out on 3 groups of rats which comprise of Group I, normal control group (normal rats fed a normal rat chow diet), Group II, diabetic control group (diabetic rats fed a normal rat chow diet), and Group III, diabetic test group (diabetic rats fed a $10 \%$ virgin coconut oil diet); each group contained 5 rats.
The animals in Group II and Group III were induced with diabetes mellitus by injecting $150 \mathrm{mg} / \mathrm{kg}$ of alloxan (Sigma) dissolved in normal saline [10] via intraperitoneal route. After 72 hours of alloxan injection, blood samples were obtained at the tail vein of the animals and the fasting blood glucose level were monitored and determined via Accu-CHEK Active glucometer. After diabetes induction, animals in each group were fed on different feed as stated above for a period of 3 weeks. However, all experiments on the animals were carried out in absolute compliance with ethical guideline for research, care, and use of laboratory animals.

2.4. Blood Sample Collection. At the end of the 3 weeks of feeding the animals, blood samples were collected from the animal through cardiac puncture into EDTA bottles after anaesthetizing the animals with chloroform. The blood samples in the bottles were centrifuge at 3000 revolutions for 10 minutes to obtain serum for lipid profile analysis.

2.5. Lipid Profile Analysis. Triglycerides (TG), total cholesterol (TC), and high density lipoprotein- (HDL-) cholesterol were evaluated with commercially available specialized kits from Randox Laboratories Limited (UK) via Jenway Spectrophotometer, Models 6405. The other lipid profiles such as very low-density lipoprotein (VLDL) and low-density lipoprotein- (LDL-) cholesterol were calculated according to Friedewald's formula [11]. VLDL-cholesterol $=$ TG/5 and LDL-cholesterol $=$ TC - VLDL-cholesterol - HDL-cholesterol.

\section{Results}

The results obtained were presented as the mean \pm standard error of mean (SEM) and analysed using analysis of variance (ANOVA) with post-hoc test (least significant differences) through Statistics Package for the Social Sciences (SPSS) version 17. The results were considered significant at $P<0.05$.

3.1. Effect of VCO on TG. As shown in Figure 1, triglycerides levels were $76.92 \pm 4.30 \mathrm{mg} / \mathrm{dL}$ for control, $95.33 \pm 3.51 \mathrm{mg} / \mathrm{dL}$ for diabetic control, and $62.83 \pm 3.38 \mathrm{mg} / \mathrm{dL}$ for diabetic rats fed a VCO diet. The value for diabetic control was significantly higher than control $(P>0.05)$ while that of diabetic rats fed a VCO diet was not significant when compared to control group.

Also, the value for diabetic rats fed a virgin coconut oil (VCO) diet was significantly lower when compared to diabetic control group $(P<0.001)$.

3.2. Effect of VCO on TC. The total cholesterol level varied among the three groups. The values were $109.05 \pm 2.09 \mathrm{mg} / \mathrm{dL}$ for control, $171.90 \pm 5.07 \mathrm{mg} / \mathrm{dL}$ for diabetic control, and $133.08 \pm 3.32 \mathrm{mg} / \mathrm{dL}$ for diabetic rats fed a VCO diet. The values for diabetic control $(P<0.001)$ and diabetic rats fed a VCO $\operatorname{diet}(P<0.01)$ were significantly higher than the control group $(P<0.001)$ as shown in Figure 2 .

However, the value of the diabetic control group was significantly higher than that of diabetic rats fed a VCO diet $(P<0.001)$. 


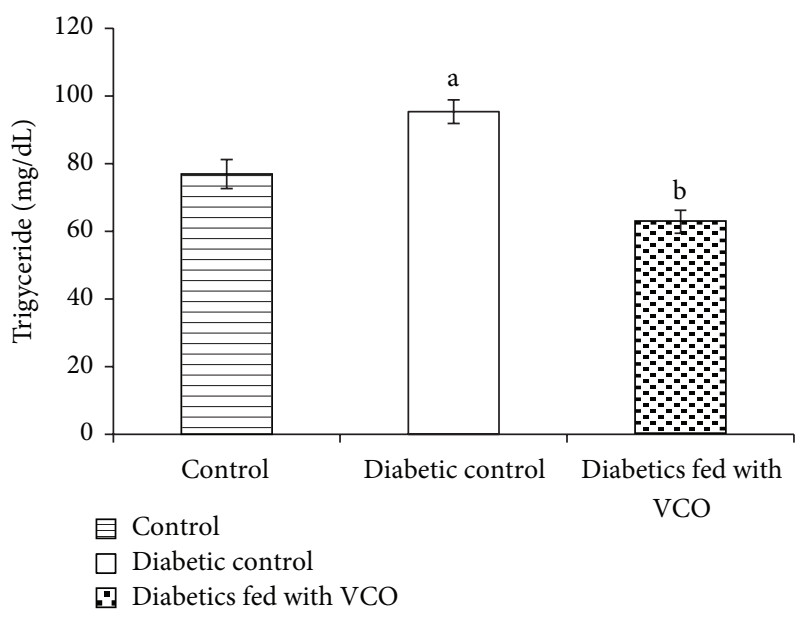

FIGURE 1: Effects of virgin coconut oil on blood triglyceride levels in diabetic rats. Bars represent the mean \pm SEM; $n=5$. ${ }^{a}$ Significantly different from control group $(P<0.05)$, ${ }^{\text {b }}$ significantly different from diabetic control group $(P<0.001)$.

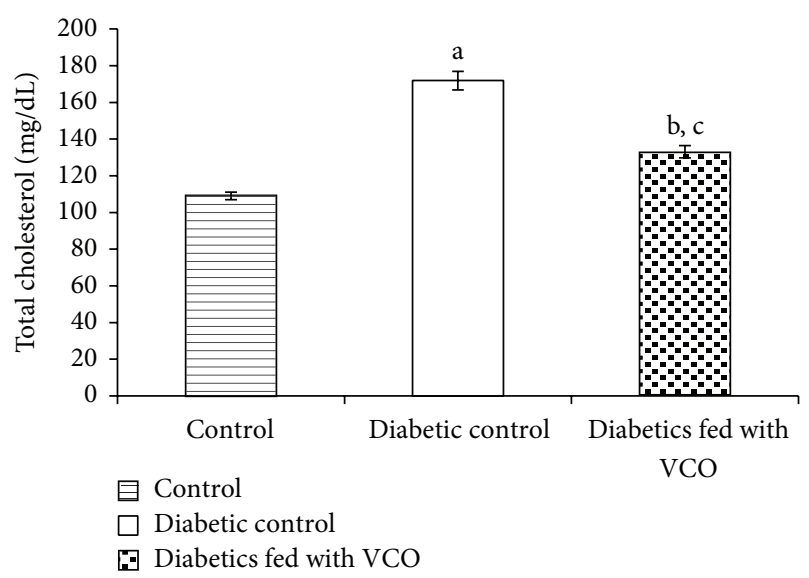

FIGURE 2: Effects of virgin coconut oil on blood total cholesterol levels in diabetic rats. Bars represent the mean \pm SEM; $n=5$. a Significantly different from control group $(P<0.001)$, ' significantly different from diabetic control group $(P<0.001)$. ' ${ }^{\mathrm{C}}$ Significantly different from control group $(P<0.01)$.

3.3. Effect of VCO on HDL. High density lipoprotein levels for control, diabetic control, and diabetics fed a VCO diet were $45.37 \pm 4.17 \mathrm{mg} / \mathrm{dL}, 28.71 \pm 3.17 \mathrm{mg} / \mathrm{dL}$, and $41.65 \pm$ $2.64 \mathrm{mg} / \mathrm{dL}$, respectively. As shown in Figure 3, HDL levels in diabetics fed a VCO diet was significant when compared with diabetic control group $(P<0.05)$ but insignificant when compared with the control group. Also, the value of the diabetic control group was significantly lower than that of the control group and diabetic rats fed a VCO diet $(P<0.05)$.

3.4. Effect of VCO on LDL. LDL levels varied among all the groups. The values for control, diabetic control, and diabetic rats fed a VCO diet were $48.30 \pm 4.67 \mathrm{mg} / \mathrm{dL}, 124.12$ $\pm 3.66 \mathrm{mg} / \mathrm{dL}$, and $78.87 \pm 5.26 \mathrm{mg} / \mathrm{dL}$, respectively. Figure 4 showed that the LDL in the plasma of the diabetic control

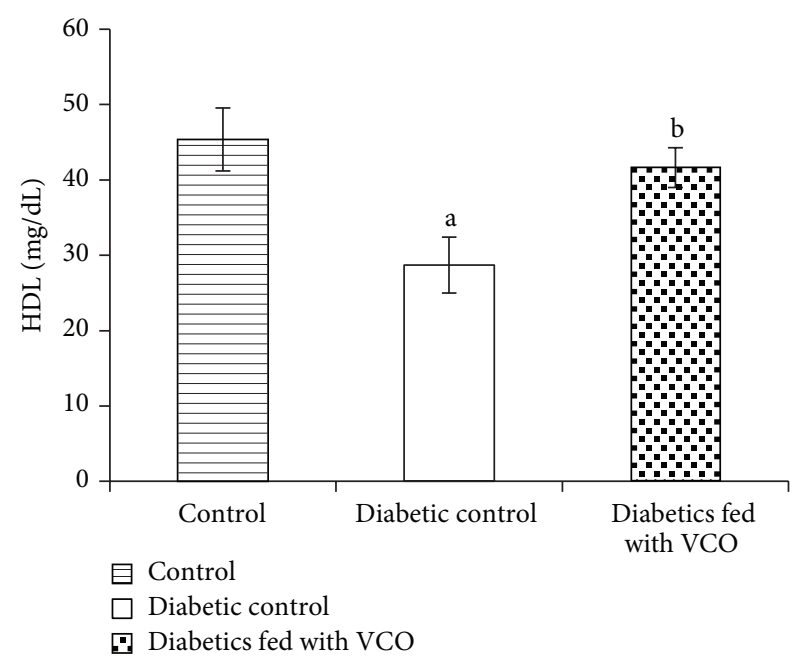

FIGURE 3: Effects of virgin coconut oil on blood high density lipoprotein levels in diabetic rats. Bars represent the mean \pm SEM; $n=5$. ${ }^{a}$ Significantly different from control group $(P<0.05)$, ${ }^{\mathrm{b}}$ significantly different from diabetic control group $(P<0.05)$.

group was significantly higher than the control group and diabetic rats fed a VCO diet $(P<0.001)$, while the plasma LDL in the diabetics fed a VCO diet was observed to be significantly higher when compared with the control group $(P<0.01)$.

3.5. Effect of VCO on VLDL. VLDL levels were $15.38 \pm$ $0.86 \mathrm{mg} / \mathrm{dL}$ for control, $19.07 \pm 0.70 \mathrm{mg} / \mathrm{dL}$ for diabetic control, and $12.57 \pm 0.67 \mathrm{mg} / \mathrm{dL}$ for diabetics fed a VCO diet (Figure 5). As shown in Figure 5, the value for diabetic control was significantly higher than the control group $(P<0.05)$ and diabetics fed a VCO diet $(P<0.001)$. However, the value of the diabetics fed a VCO diet was insignificant when compared with the control group.

\section{Discussion}

Lipids, even though important to the body, can be harmful when above normal. Researches had revealed that people within the ages of 40 and above are prone to cardiovascular disorder which arises from metabolic derangements (such as diabetes mellitus) affecting carbohydrates and proteins $[12,13]$. Hence, regular determination of lipid levels, proper dieting is paramount in preventing the prevalence of certain risk factors associated with diabetes mellitus.

From the result of this study, it was evident that there was significant increase in the concentration of TG, TC, LDL, and VLDL, while there was low level of HDL in diabetic control group when compared to the control group. This increase in TG, TC, LDL, and VLDL and decrease in HDL denotes an increase in the risk of cardiovascular diseases associated with diabetes mellitus.

However, it was observed that there was a decrease in the concentration of TG, TC, LDL, and VLDL, while there was an increase in the level of HDL in diabetics fed a VCO diet when compared to diabetic control group. Therefore, it 


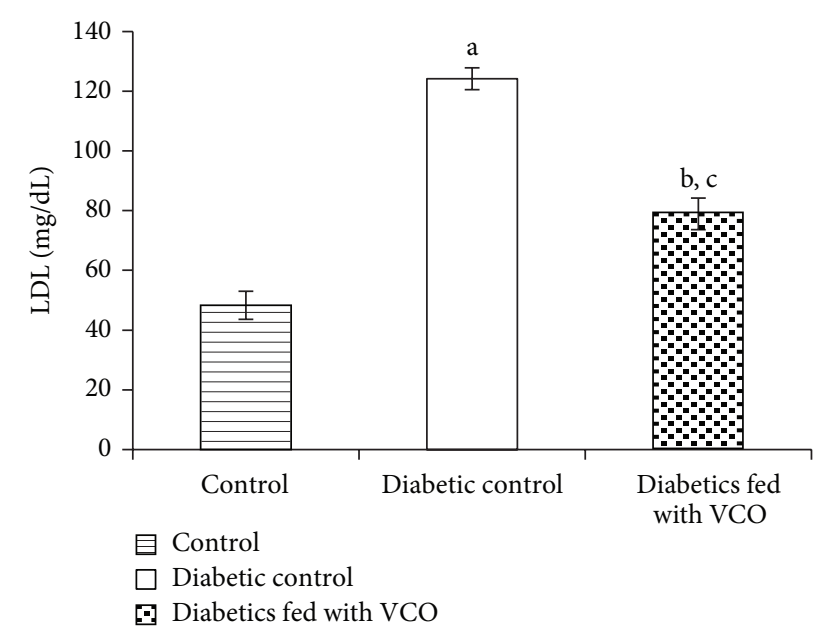

FIGURE 4: Effects of virgin coconut oil on blood low density lipoprotein levels in diabetic rats. Bars represent the mean \pm SEM; $n=5$. ${ }^{a}$ Significantly different from control group $(P<0.001)$, ${ }^{\mathrm{b}}$ significantly different from diabetic control group $(P<0.001)$. ${ }^{\mathrm{c}}$ Significantly different from control group $(P<0.01)$.

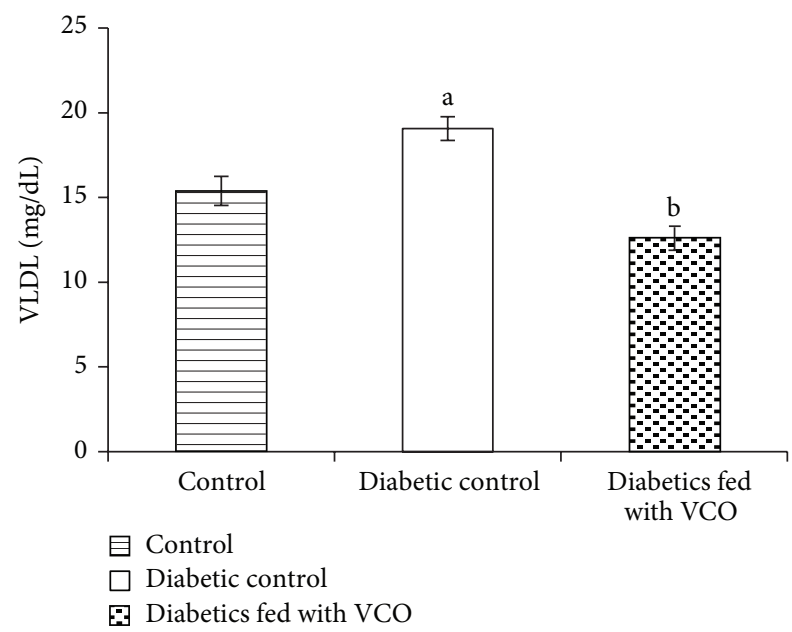

FIGURE 5: Effects of virgin coconut oil on blood very low density lipoprotein levels in diabetic rats. Bars represent the mean \pm SEM; $n=5$. 'Significantly different from control group $(P<0.05)$, ${ }^{\mathrm{b}}$ significantly different from diabetic control group $(P<0.001)$.

is not unreasonable to suggest that virgin coconut oil (VCO) is therapeutic. The results of this study agree with previous studies which reported that ingestion of virgin coconut oil as a whole is antiatherogenic and hypocholesterolemic $[9,14]$.

The reduction in the levels of TG, TC, LDL, and VLDL by virgin coconut oil which in excess possess risks of ailments and diseases is enough reason to claim that VCO exerts a protective effect in the management of diabetes mellitus in addition to preventing cardiovascular disease. However, the significant increase in HDL level in diabetics fed a VCO diet when compared to diabetics control group is considered protective against chronic heart diseases. One mechanism for this is the fact that HDL plays a vital role in reversing cholesterol transport from extra hepatic tissues to the liver for onward excretion in bile. Thus, reducing plasma cholesterol levels [3].

Moreover, a number of studies have suggested that reduced LDL and TC but increased HDL levels have improved survival and prevented heart attacks in people with or without heart diseases $[3,6]$. Therefore, since increased LDL level which causes atherosclerosis that results to cardiovascular diseases in diabetics is seen to have been reduced by VCO with a significant increase in the HDL, it can be said that VCO is medicinal. However, VCO is a mixture of several biochemicals, including medium chain fatty acids, lauric acid, caprylic acid, and myristic acid, whose efficacy may have resulted from the therapeutic and antioxidant effects of these compounds.

\section{Conclusion}

Hence, it can therefore be deduced that dietary consumption of virgin coconut oil ( $\mathrm{VCO}$ ) ameliorates lipid profiles in diabetes mellitus.

\section{Conflict of Interests}

The authors declare that there is no conflict of interests regarding the publication of this paper.

\section{References}

[1] W. F. Boron and E. L. Boulpaep, Medical Physiology: A Cellular and Molecular Approach, Elsevier Saunders, Philadelphia, Pa, USA, 2005.

[2] "Hope 4 Diabetes," http://www.hope4diabetes.info/generalinformation/diabetes-a-worldwide-epidemic.html.

[3] R. K. Murray, D. A. Bender, K. M. Botham, P. J. Kennelly, V. W. Rodwell, and P. A. Weil, Harper's Illustrated Biochemistry, McGraw-Hill, San Francisco, Calif, USA, 28th edition, 2009.

[4] K. G. Nevin and T. Rajamohan, "Beneficial effects of virgin coconut oil on lipid parameters and in vitro LDL oxidation," Clinical Biochemistry, vol. 37, no. 9, pp. 830-835, 2004.

[5] B. M. Kochikuzhyil, K. Devi, and S. R. Fattepur, "Effect of saturated fatty acid-rich dietary vegetable oils on lipid profile, antioxidant enzymes and glucose tolerance in diabetic rats," Indian Journal of Pharmacology, vol. 42, no. 3, pp. 142-145, 2010.

[6] R. Lee and M. J. Balick, "Palms, people, and health," Explore, vol. 4, no. 1, pp. 59-62, 2008.

[7] S. Schaffer, W. E. Müller, and G. P. Eckert, "Tocotrienols: constitutional effects in aging and disease," The Journal of Nutrition, vol. 135, no. 2, pp. 151-154, 2005.

[8] J. Kamsiah, S. N. Aziz, S. T. Siew, and I. S. Zahir, "Changes in serum lipid profile and malondialdehyde following the consumption of fresh or heated red palm oil," Medical Journal of Islamic Academy of Sciences, vol. 14, no. 2, pp. 79-86, 2001.

[9] M. I. Akpanabiatu, I. B. Umoh, E. O. Udosen, A. E. Udoh, and E. E. Edet, "Rat serum electrolytes, lipid profile and cardiovascular activity on Nauclea latifolia leaf extract administration," Indian Journal of Clinical Biochemistry, vol. 20, no. 2, pp. 29-34, 2005.

[10] T. Szkudelski, "The mechanism of alloxan and streptozotocin action in B cells of the rat pancreas," Physiological Research, vol. 50, no. 6, pp. 537-546, 2001. 
[11] W. T. Friedewald, R. I. Levy, and D. S. Fredrickson, "Estimation of the concentration of low-density lipoprotein cholesterol in plasma, without use of the preparative ultracentrifuge," Clinical Chemistry, vol. 18, no. 6, pp. 499-502, 1972.

[12] O. P. Agarwal, "Prevention of atheromatous heart disease," Angiology, vol. 36, no. 8, pp. 485-492, 1985.

[13] H.-Y. Li, G.-X. Pang, and Z.-Z. Xu, "Tear film function of patients with type 2 diabetes," Acta Academiae Medicinae Sinicae., vol. 26, no. 6, pp. 682-686, 2004.

[14] S. Arunima and T. Rajamohan, "Virgin coconut oil improves hepatic lipid metabolism in rats-compared with copra oil, olive oil and sunflower oil," Indian Journal of Experimental Biology, vol. 50, no. 11, pp. 802-809, 2012. 

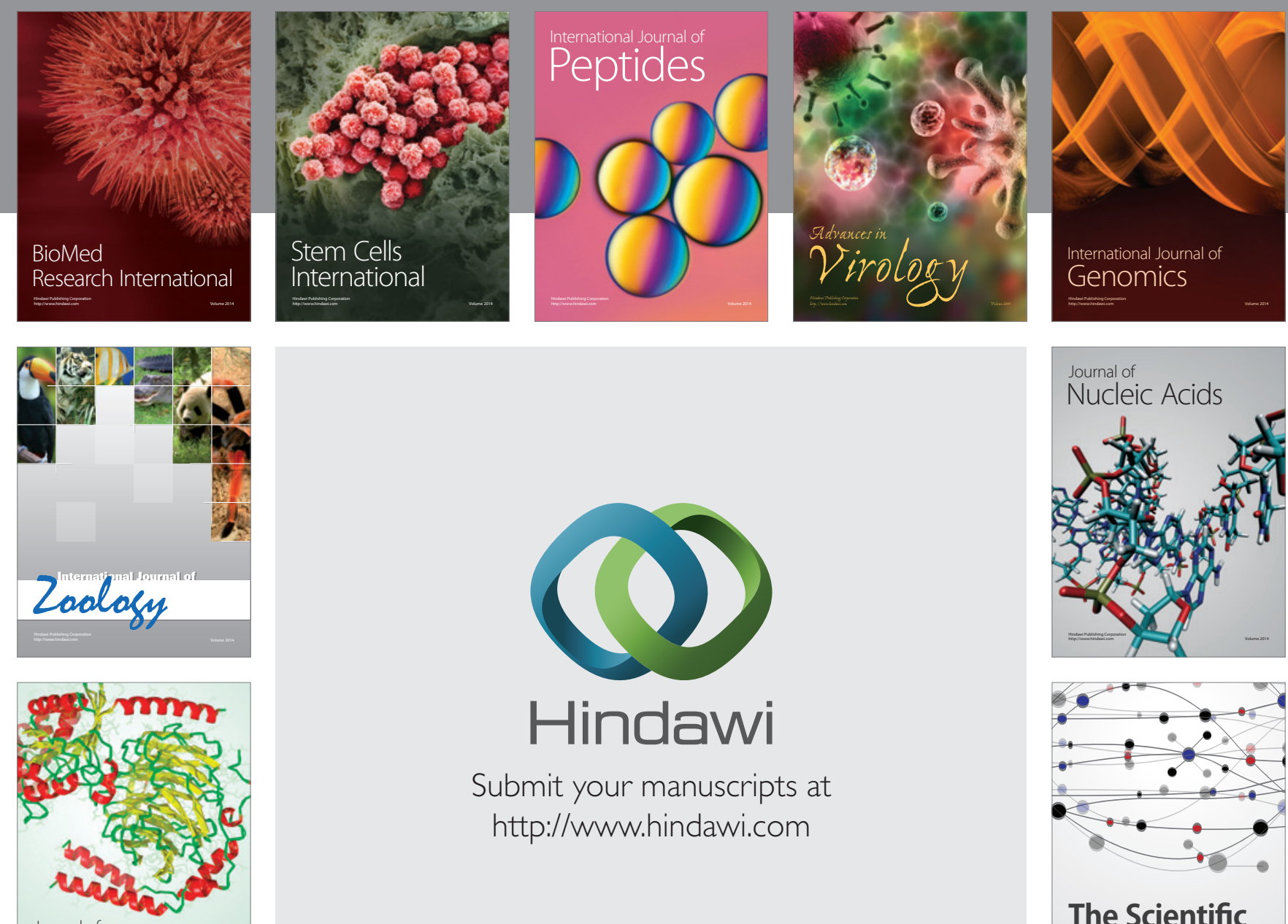

Submit your manuscripts at

http://www.hindawi.com

Journal of
Signal Transduction
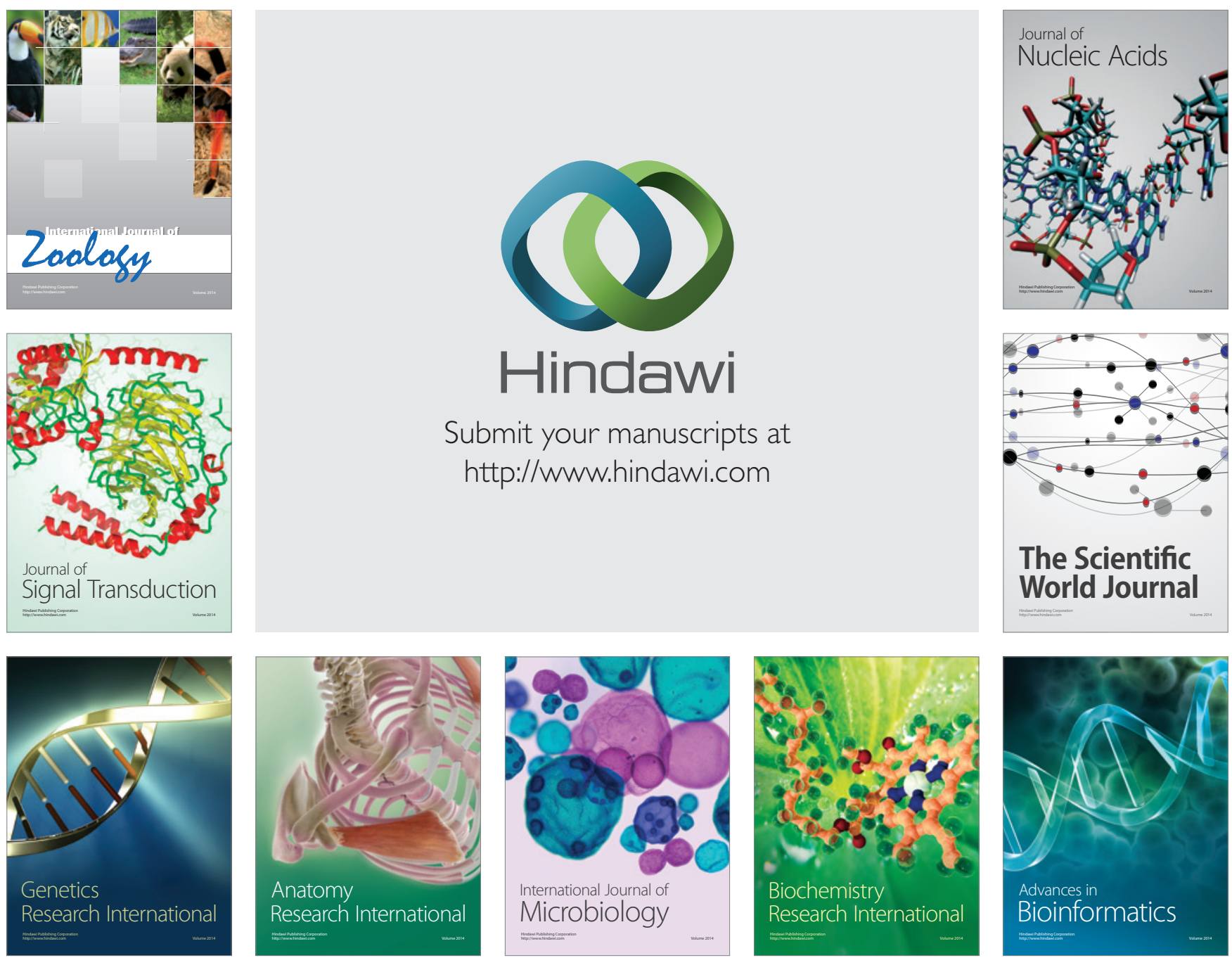

The Scientific World Journal
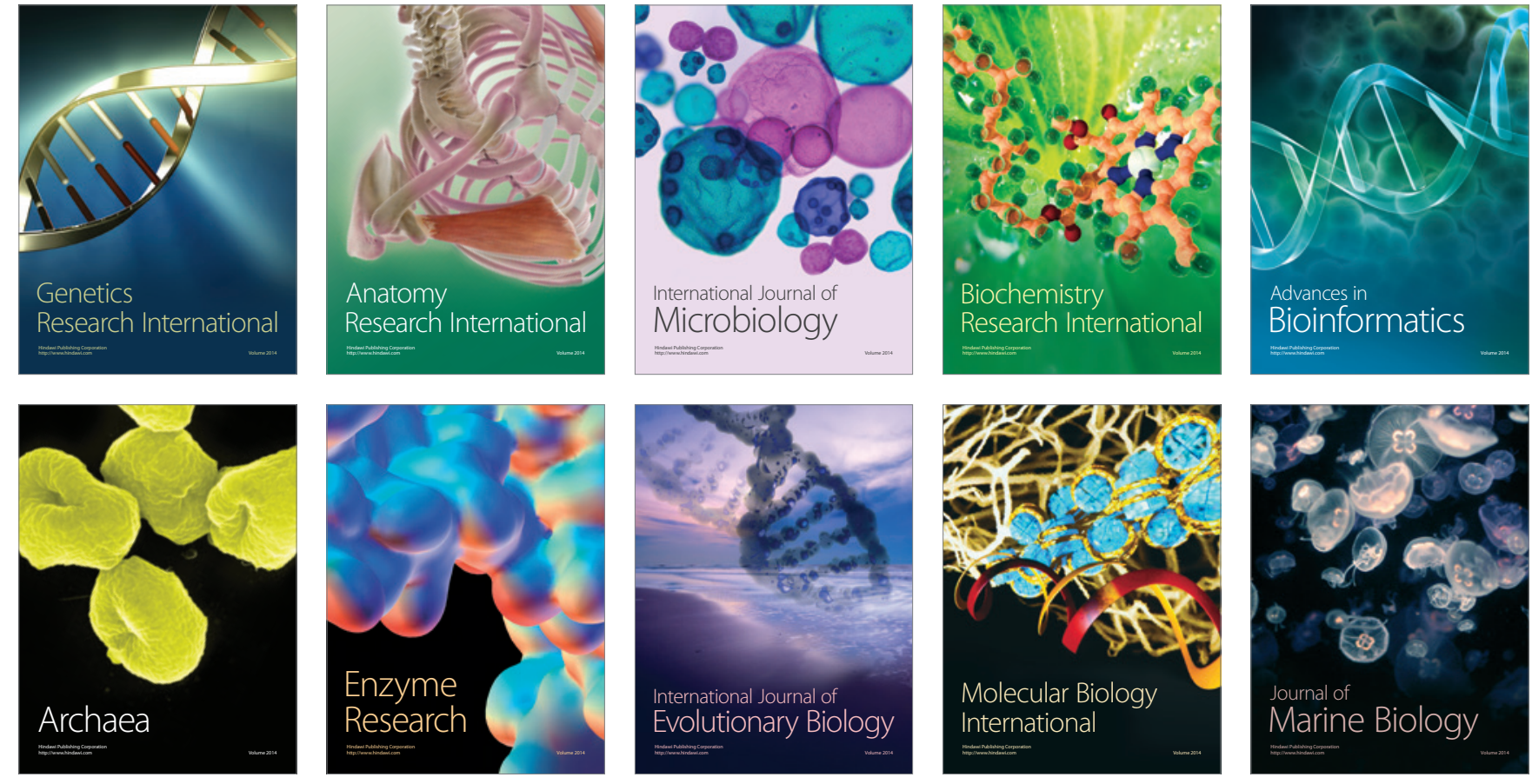